identities in maternal mental health research. Using a cumulative or additive measure of MFD might underestimate the association between discrimination and PPD specifically in minority and immigrant mothers who face MFD. Health care providers should consider the effects of MFD on PPD among mothers, especially for women located at more than one marginalized axis of identity.

\section{RF37 PREDICTORS OF ATTITUDES TOWARDS PEOPLE WITH DISABILITY IN AUSTRALIA: FINDINGS FROM A CROSS-SECTIONAL SURVEY OF AUSTRALIAN ADULTS}

A Kavanagh*, AM Bollier, L Krnjacki, G Katsikis, V Kasidis, J Ozge, A Milner. Melbourne School of Population and Global Health, University of Melbourne, Melbourne, Australia

10.1136/jech-2019-SSMabstracts. 152

Background There have been many studies on attitudes to disability, but few are nationally representative, and little is known about how attitudes vary by socio-demographic characteristics and contact with disabled people.

Methods We used data from 2,000 members of an Australian probability panel who completed a survey on disabilityrelated attitudes. Attitudes were measured with the Attitudes to Disability Scale (ADS), which asks 16 questions in 5point Likert-type format. The outcome for this analysis was the Prospects sub-scale, which asks whether the capacities and aspirations of disabled people should differ from those of non-disabled people. Raw scores out of 20 points were standardized (mean 0, SD 1) with positive scores representing more positive attitudes. Sociodemographic variables (disability/carer status, age cohort, education level, country of birth, socio-economic disadvantage, gender, and region of residence) were fitted in a linear regression model to examine predictors of attitudes. Eight measures of lifetime contact with people with disability (e.g. ever lived with a person with disability [yes/no]) were then fitted in separate models to examine associations with attitudes, adjusting for potential confounders excludes participants with a disability and carers.

Results Female (versus male) gender predicted more positive attitudes $(\beta=0.36,95 \%$ CI $0.23,0.49)$, as did overseas English-speaking origin $(\beta=0.20,95 \%$ CI $0.02,0.39)$ relative to native Australian, and year 12 educational attainment $(\beta=0.41,95 \%$ CI $0.21,0.60)$ or university study $(\beta=0.39$, $95 \%$ CI $0.20,0.58)$ relative to less than year 12 . People with disability (versus non-disabled non-carers) had more negative attitudes $(\beta=-0.15,95 \%$ CI $-0.30,-0.01)$, as did members of the oldest (Lucky) generation versus the youngest (iGeneration) ( $\beta=-0.37,95 \%$ CI $-0.62,-0.12)$ and overseas non-English speaking origin $(\beta=-0.37,95 \%$ CI -0.62 , -0.13) versus native Australian. In the 8 contact models, 4 were associated with more positive attitudes: ever having a colleague $(\beta=0.29,95 \%$ CI $0.13,0.45)$; classmate $(\beta=0.26$, $95 \%$ CI $0.09,0.43)$; close friend $(\beta=0.25,95 \%$ CI 0.07 , $0.42)$; and teacher/boss $(\beta=0.27,95 \%$ CI $0.02,0.52)$ with disability.

Conclusion This study is the first Australian population-based study of predictors of attitudes to disability. Internalized stigma might explain why disability was associated with more negative attitudes among disabled people. Because this is a cross-sectional study, we do not know the direction of the relationship between having friends with disability and positive attitudes however our findings suggest that peer relationships in social environments and contact in work and educational settings promote positive attitudes to disabled people.

\section{Poster Programme}

\section{P01 CHILDHOOD SOCIO-ECONOMIC POSITION AND MIDLIFE PHYSICAL FUNCTIONING: EXPLORING ASSOCIATIONS AND UNDERLYING MECHANISMS IN THE 1958 BRITISH BIRTH COHORT}

A Ahmed* , SP Pereira. Epidemiology and Public Health, University College London, London, UK

10.1136/jech-2019-SSMabstracts. 153

Background Evidence suggests that childhood socioeconomic position (CSEP) is associated with adult physical functioning $(\mathrm{PF})$. However, literature is limited on key life-course intermediaries that explain this relationship. In the 1958 British birth cohort, we aimed to examine (1) the association between CSEP and PF in midlife, and (2) potential adult intermediaries (i.e. social, psychological, behavioural and biological pathways) that could explain the CSEP-PF relationship.

Methods CSEP recorded at birth (or at 7y) was represented by 4 categories of father's occupational class (professional/ managerial, skilled non-manual, skilled manual and partly skilled/unskilled). PF was assessed at $50 \mathrm{y}$ using the validated PF subscale of the SF-36 questionnaire. Participants with a score at or below the gender-specific 10th-centile were classified as having poor PF. Prospectively collected data represented each of the four potential intermediary pathways: social (42y socioeconomic position, 33y education), psychological (42y mental health), behavioural (42y smoking, drinking and physical activity) and biological (45y measured BMI, waist-hip ratio, blood pressure, lung function, HbA1c and blood lipids). Gender specific associations were examined using logistic regression, in STATA v15. Adjustments were made for prospectively recorded early-life covariates (birthweight, maternal smoking, childhood disability and presence of childhood illnesses).

Results In unadjusted analysis ( $\mathrm{n}=4056$ men, $\mathrm{n}=4375$ women) odds ratios (ORs) for poor PF increased with decreasing CSEP. This trend was maintained after covariate adjustment e. g. in women, compared to the professional/managerial class, adjusted-OR for skilled non-manual was 1.31[0.77,2.24]; skilled manual was $1.86[1.28,2.69]$ and partly skilled/unskilled was 2.23[1.49,3.35]. After accounting for the social pathway, associations were markedly attenuated, particularly in women; e.g. compared to the professional/managerial class OR for skilled non-manual was $0.87[0.46,1.68]$; skilled manual was $1.24[0.80,1.93]$ and partly skilled/unskilled was 1.30 $[0.79,2.14]$. Behavioural factors attenuated associations to a lesser extent. Biological factors completely attenuated associations in women, whereas associations remained in men. Associations were little affected by psychological factors.

Conclusion While there was a trend between lower CSEP and higher risk of poor midlife PF in men and women, intermediary pathways differed by gender. Better adult education and employment opportunities may help alleviate the burden of low CSEP for all, however improving biomedical profiles may 
have greater benefits for women. Study strengths include the large nationally representative cohort followed from birth and prospective measures of CSEP, PF and covariates. However, the current examination based on a complete case analysis, disregards missing data, which will be addressed in future work.

\section{P02 DOES ALL HEALTH SERVICES AND DELIVERY RESEARCH (HSDR) GET PUBLISHED? A STUDY TO FOLLOW UP THE PUBLICATION STATUS OF COHORTS OF HSDR}

${ }^{1} \mathrm{AA}$ Ayorinde* ${ }^{2}{ }^{2}$ Williams, ${ }^{2} \mathrm{R}$ Mannion, ${ }^{3} \mathrm{~F}$ Song, ${ }^{4} \mathrm{M}$ Skrybant, ${ }^{1} \mathrm{SRJ}$ Lilford, ${ }^{1} \mathrm{YF}$ Chen ${ }^{1}$ Warwick Medical School, University of Warwick, Coventry, UK; ${ }^{2}$ Health Services Management Centre, University of Birmingham, Birmingham, UK; ${ }^{3}$ Norwich Medical School, University of East Anglia, Norwich, UK; ${ }^{4}$ Institute of Applied Health Research, University of Birmingham, Birmingham, UK

\subsection{6/jech-2019-SSMabstracts. 154}

Background Publication bias has been demonstrated in several high-profile cases associated with clinical trials of pharmaceuticals, but empirical evidence on this bias is scarce in HSDR, which concerns research to produce evidence on the quality, accessibility and organisation of health services. We set out to gauge the existence and scale of publication bias in quantitative HSDR by following up the publication status of four cohorts of studies identified from registries and conferences.

Methods We identified HSDR cohorts from four sources: (1) all quantitative studies funded by the NIHR HS\&DR Programme (and its predecessors) between 2007-2014 ( $n=100)$; (2) a random sample of quantitative HSDR studies registered with HSRProj (a US-based prospective registry of health service and public health research) and completed in 2012 $(n=100)$; (3) abstracts from the 2012 International Society for Quality in Health Care (ISQua) conference $(n=50)$ and (4) Health Services Research UK (HSRUK) conference, 2012-14 $(n=50)$. We checked the publication status of selected studies in 2018 by searching PubMed and Google. When no relevant publications were found, we contacted the investigators to verify the publication status. We compiled descriptive statistics for each cohort and used univariate analysis to explore the association between reporting of statistically significant findings and publication status.

Results Following online search of publications, we needed to contact investigators for $145 / 300(48 \%)$ of the studies and had a response rate of $60 \%(67 / 111)$ among those with valid contact details. Publication of findings in academic journals varies between cohorts: NIHR 64\%, HSRProj 75\%, ISQua $26 \%$ and HSRUK 70\%. When grey literature (including technical reports available online) was taken into account, the publication rates were NIHR 100\%, HSRProj 91\%, ISQua $32 \%$ and HSRUK $80 \%$. Statistically significant findings were reported by $79 \%(237 / 300)$ of the studies. There was no apparent association between reporting of statistically significant findings and publication in academic journals for the combined NIHR/HSRProj cohort (OR 1.19, 0.50-2.81) and conference cohort (OR 0.88, 0.25-3.10).

Discussion The rates of publication were generally high among HSDR studies that we sampled (which are likely to represent the high rectitude end of distribution), except in the ISQua cohort where the originators of the studies tended to be service providers rather than academic researchers. The power of our exploratory analysis was limited by the relatively small number of studies reporting non-significant findings and/or having a non-publication status. The publication policy of research funders (e.g. NIHR) appears to be effective in ensuring the publication of research findings.

\section{P03 A SYSTEMATIC REVIEW OF INTERVENTIONS IN COMMUNITY INFRASTRUCTURE (PLACES AND SPACES) TO BOOST SOCIAL RELATIONS AND COMMUNITY WELLBEING}

${ }^{1} \mathrm{AM}$ Bagnall ${ }^{*}$, 'J South, ${ }^{1} \mathrm{~K}$ Southby, ${ }^{1} \mathrm{~S}$ Di Martino, ${ }^{2} \mathrm{G}$ Pilkington, ${ }^{3} \mathrm{~B}$ Mitchell, ${ }^{2} \mathrm{~A}$ Pennington, ${ }^{2} \mathrm{R}$ Corcoran. ${ }^{1}$ School of Health and Community Studies, Leeds Beckett University, Leeds, UK; ${ }^{2}$ Institute of Psychology, Health and Society, University of Liverpool, Liverpool, UK; ${ }^{3}$ Leeds Business School, Leeds Beckett University, Leeds, UK

\subsection{6/jech-2019-SSMabstracts. 155}

Background Stakeholder engagement for the What Works Centre for Wellbeing's (WWCW) Community Wellbeing Evidence Programme identified 'boosting social relations' in communities as a priority policy-related topic. A scoping review of 34 reviews identified evidence gaps relating to social relations in the following areas: community infrastructure (places and spaces); interventions to reduce or prevent social isolation in adults $<60$ years; community engagement and volunteering; social network analyses. We developed 'community infrastructure (places and spaces)' as a systematic review, as this can be addressed at a local or regional level and has potential to produce immediate practical impact.

Methods We searched 11 bibliographic databases from 19972017, performed reference and citation checking, searched the websites of relevant organisations, and issued a call for evidence through the WWCW. We included studies which reported: interventions to improve or make alternative use of physical places and spaces at community or neighbourhood level; outcomes of social relations, community wellbeing and related concepts; quantitative, qualitative and mixed methods studies, and process evaluations. Two reviewers undertook study selection. One reviewer undertook data extraction and validity assessment, with a random $20 \%$ checked by a second reviewer. Validity of included studies was assessed using established checklists. Following thematic synthesis of qualitative data, a narrative synthesis was produced for each of eight intervention categories. The GRADE and CERQual approaches were used to rate the overall strength of evidence for each outcome.

Results 51 studies, mostly of poor to moderate quality, were included. The better quality evidence was qualitative, and most of the review's findings come from the thematic synthesis of qualitative evidence

There was moderate evidence that

i. Community hubs may promote social cohesion, increase social capital and build trust between people, widen social networks and increase interaction between people, and increase people's knowledge or skills; 